\title{
Singular kernel problems in materials with memory
}

\section{Sandra Carillo}

\section{Meccanica}

An International Journal of Theoretical and Applied Mechanics AIMETA

ISSN 0025-6455

Meccanica

DOI 10.1007/s11012-014-0083-y

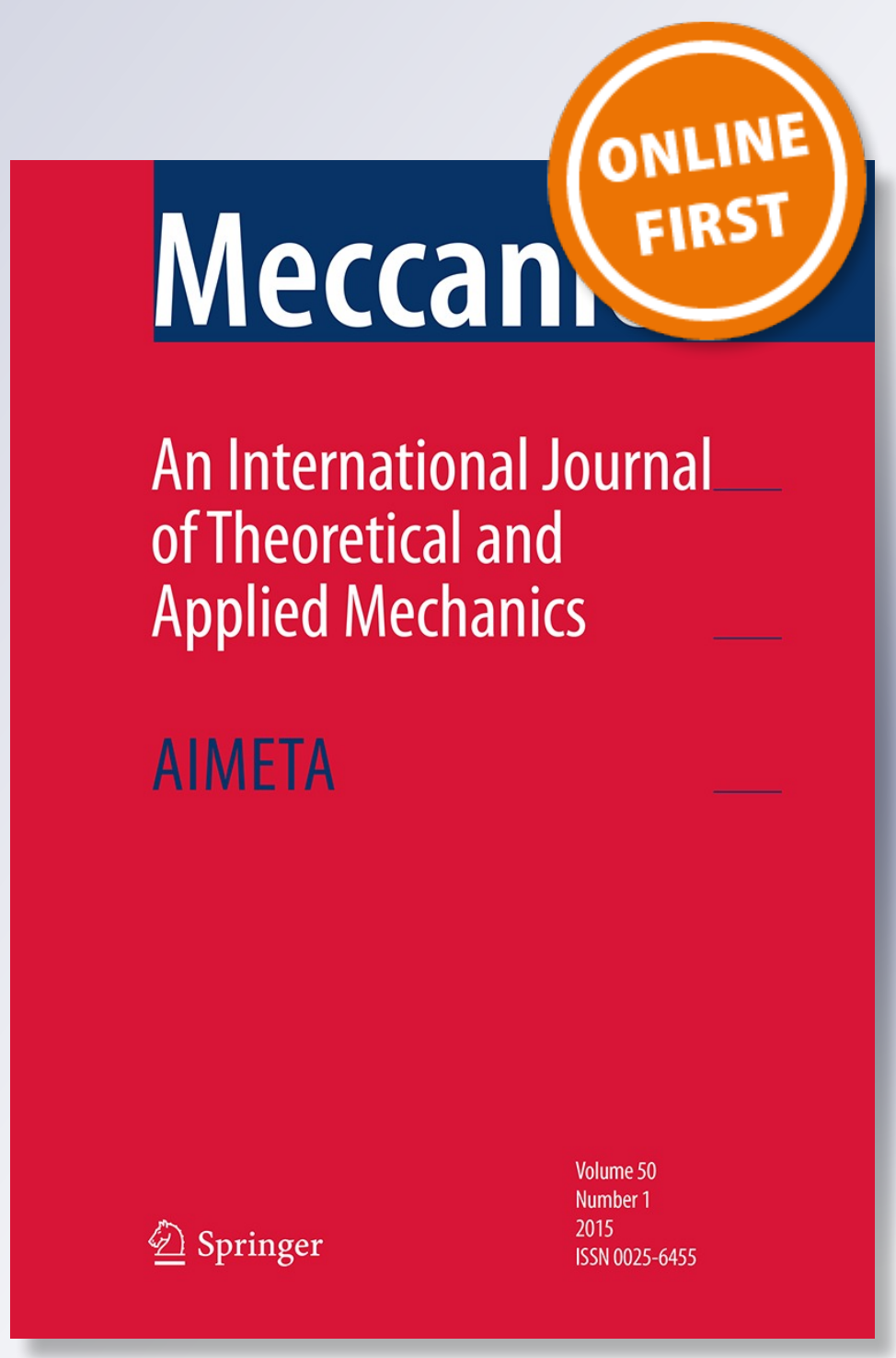

算 Springer 
Your article is protected by copyright and all rights are held exclusively by Springer Science +Business Media Dordrecht. This e-offprint is for personal use only and shall not be selfarchived in electronic repositories. If you wish to self-archive your article, please use the accepted manuscript version for posting on your own website. You may further deposit the accepted manuscript version in any repository, provided it is only made publicly available 12 months after official publication or later and provided acknowledgement is given to the original source of publication and a link is inserted to the published article on Springer's website. The link must be accompanied by the following text: "The final publication is available at link.springer.com". 


\title{
Singular kernel problems in materials with memory
}

\author{
Sandra Carillo
}

Received: 14 April 2014/ Accepted: 24 November 2014

(C) Springer Science+Business Media Dordrecht 2014

\begin{abstract}
In recent years the interest on devising and study new materials is growing since they are widely used in different applications which go from rheology to bio-materials or aerospace applications. In this framework, there is also a growing interest in understanding the behaviour of materials with memory, here considered. The name of the model aims to emphasize that the behaviour of such materials crucially depends on time not only through the present time but also through the past history. Under the analytical point of view, this corresponds to model problems represented by integro-differential equations which exhibit a kernel non local in time. This is the case of rigid thermodynamics with memory as well as of isothermal viscoelasticity; in the two different models the kernel represents, in turn, the heat flux relaxation function and the relaxation modulus. In dealing with classical materials with memory these kernels are regular function of both the present time as well as the past history. Aiming to study new materials integrodifferential problems admitting singular kernels are compared. Specifically, on one side the temperature evolution in a rigid heat conductor with memory characterized by a heat flux relaxation function
\end{abstract}

S. Carillo $(\bowtie)$

Dipartimento di Scienze di Base e Applicate per

l'Ingegneria - Sez. MATEMATICA, SAPIENZA

Università di Roma, Via A. Scarpa 16, 00161 Rome, Italy

e-mail: sandra.carillo@sbai.uniroma1.it;

sandra.carillo@uniroma1.it singular at the origin, and, on the other, the displacement evolution within a viscoelastic model characterized by a relaxation modulus which is unbounded at the origin, are considered. One dimensional problems are examined; indeed, even if the results are valid also in three dimensional general cases, here the attention is focussed on pointing out analogies between the two different materials with memory under investigation. Notably, the method adopted has a wider interest since it can be applied in the cases of other evolution problems which are modeled by analogue integrodifferential equations. An initial boundary value problem with homogenous Neumann boundary conditions is studied.

Keywords Viscoelasticity - Thermodynamics with memory $\cdot$ Integro-differential equations $\cdot$ Singular relaxation modulus $\cdot$ Singular kernel $\cdot$ Singular heat flux relaxation function

\section{Introduction}

The models here considered are well known ones and refer to materials with memory as they are termed in the wide literature which is concerned about their physical (thermodynamical and/or mechanical) behaviours, on one side, and the many interesting analytical problems, on the other one. Specifically, in the case of a viscoelastic body, its deformation does 
not depend only on the mechanical status of the body in the present time, but, also, on its deformation history according to the well known model [1-3]. Viscoelastic components are more and more often used in devising new and smart materials in general. The interest in this subject is testified by many books and Conferences such as such as [4-7] which may be considered as sample ones; the first two are very recent books providing an overview on the subject aiming, the first one, to viscoelasticity models referring to study earthquakes and the second one to an updated overview on fractional calculus in linear viscoelasticity. Indeed, according to Fabrizio [8], who, in 2014, further to cite experimental results, analyzes the connection between Volterra and fractional derivatives models, the growing interest in models based on fractional derivatives is also due to the need to devise new tools to study materials whose behaviour cannot be described when the classical regularity hypothesis on the kernel in the integro-differential equations are assumed. This is the case studied, again in 2014, by Deseri et al. [9], who show how a fractional derivative model can be adapted to describe bio-materials. Indeed, the Special Issue of Discrete and Continuous Dynamical Systems-Series B dedicated to Mauro Fabrizio [10], comprises articles dedicated to materials with memory or which may be termed new, such as [11-24] and mathematical models to describe the behaviour of problems which arise in biological contexts [25-28]. These results motivate us to adopt, in this present article, less restrictive functional requirements on the kernel.

The two books [6, 7] are Special Volumes which comprise results presented in Conferences devoted to new and smart materials together with analytical problems arising from the investigation of such models; these books are listed here as examples of the current interest in materials with memory and in the related mathematical models.

The general regularity assumptions on the relaxation modulus guarantee the solution existence and uniqueness of Volterra type problems, as pointed out by many authors and firstly proved by Dafermos [29, 30]. Nevertheless, the idea of singular kernels to model particular cases of viscoelastic behaviours was introduced by Boltzmann [31] in the nineteenth century. Later, the same model was further investigated, since the middle of twentieth century, by Zimm et al. [1, 32-34] referring to polymers. On the other hand, many authors $[9,35-38]$ pointed out also the applicative interest of new polymers and/or biomaterials whose mechanical response is not modeled by a Volterra type integro-differential equation with a regular kernel. A wide research activity is testified by many references such as [39-46] to mention some of those ones concerning singular kernel problems both under the analytical as well as the model point of view. Furthermore, other authors, such as Berti [47] and Grasselli and Lorenzi [48] study viscoelasticity problems exhibiting a singular memory kernel. The thermodynamical admissibility of a singular viscoelastic model characterized by a singular viscoelastic relaxation modulus is analyzed by Giorgi and Morro [49]. The references [50-58] are all concerning singular kernel problems both in rigid thermodynamics with memory as well as in viscoelasticity. Specifically, [50-52, 57, 58] study asymptotic behaviour of solutions.

In this framework, the study here presented are part of a wide research project concerning the mechanical behavior of materials with memory, in which the author is involved. Thus, the aim here is to further develop results obtained in joint research works with Valente and Vergara Caffarelli [59-63]. Here, the attention is focussed on the existence and uniqueness of the solution admitted by singular kernel problems in materials with memory, previous and in progress results are comprised in [61-63]. Indeed, this study refers to Neumann boundary conditions while previous results were concerned about Dirichlet boundary conditions.

The material is organized as follows. The opening Sect. 2 is concerned about the physical model. Crucial assumptions which characterize, in turn, the model of rigid heat conduction with memory and of isothermal viscoelasticity are comprised in the two sub-Sections.

The key references, wherein the aspects of the models here of interest are given, are [64, 65] concerning rigid heat conduction and $[2,3]$ in the case of isothermal viscoelasticity. Notably, as pointed out in [66], the analogous functional spaces, wherein the solutions of the evolution problems are looked for, are obtained in the cases of the two different models. Indeed, throughout the whole article, the two different models are compared in connection to the singular kernel problems under investigation. In particular, analogies between the two models of materials with memory both under the physical as well as under the 
functional spaces point of view are shown in [66], here the comparison is extended to singular kernel problems which were not considered previously. In particular, the analogy remains valid when the regularity requirement of the kernel is relaxed to consider also the case when, in turn, the heat flux relaxation function and the relaxation modulus are unbounded at the origin provided they are integrable $\left(L^{1}\right)$.

The next Sections are concerned about evolution problems: Sects. 3 and 4, respectively, study evolution problems in heat conduction with memory and in isothermal viscoelasticity. Each Section is divided in two parts; the first one dedicated to classical regular problems, and, then, the second one, devoted to singular problems. Specifically, here singular problems with assigned initial and Neumann homogeneous boundary conditions are considered. In the subsequent Sect. 5 existence and uniqueness results are given. In the closing Sect. 6 some perspective problems and current investigations are mentioned. Detailed proofs of results previously stated are included in the Appendix.

\section{The model of material with memory}

In this Section some of the key features of the model of a material with memory are recalled referring to the two cases under consideration, namely rigid heat conduction with memory and isothermal viscoelasticity.

\subsection{Rigid heat conduction with memory}

Here the model of a rigid heat conductor with memory, restricting only to a description of the physical assumptions and to some properties needed in the following, is briefly recalled. First of all, let $\Omega \subset \mathbb{R}^{3}$ denote the body configuration, the main assumptions on the rigid heat conductor with memory are [64]:

- $\Omega$ is a connected set with a smooth boundary;

- $\Omega$ changes its thermodynamical status according to linear heat conduction with memory; that is, it depends on time via present and past times, i.e., on the thermal history of the material;

- The environment is assumed not to be affected by the presence of the body itself;
- no space dependence, i.e., $\mathbf{x}$-dependence is omitted under the assumption that the material is homogeneous and isotropous.

In particular, the approach presented by Fabrizio et al. [64], and, subsequently, in [65] is adopted. The internal energy $e$ is assumed to be linearly related to the relative temperature $u:=\theta-\theta_{0}$, where $\theta_{0}$ denotes a fixed reference temperature, namely

$e(t)=\alpha_{0} u(t)$.

The heat flux $\mathbf{q} \in \mathbb{R}^{3}$, when, in turn, $\mathbf{g}:=\nabla u$ denotes the temperature-gradient, and

$\overline{\mathbf{g}}^{t}(\tau)=\int_{t-\tau}^{t} \mathbf{g}(s) d s$

the integrated history of the temperature-gradient, reads

$$
\begin{aligned}
& \mathbf{q}(t)=-\int_{0}^{\infty} k(\tau) \mathbf{g}(t-\tau) d \tau \quad \text { or } \\
& \mathbf{q}(t)=\int_{0}^{\infty} \dot{k}(\tau) \overline{\mathbf{g}}^{t}(\tau) d \tau .
\end{aligned}
$$

The heat flux relaxation function $k(t)$, in (3), is given by

$k(t)=k_{0}+\int_{0}^{t} \dot{k}(s) d s$,

where $k_{0} \equiv k(0)$ denotes the initial heat flux relaxation coefficient, that is the initial (positive) value assumed by the heat flux relaxation function. It is further required that

$\dot{k} \in L^{1}\left(\mathbb{R}^{+}\right) \cap L^{2}\left(\mathbb{R}^{+}\right) \quad$ and $\quad k \in L^{1}\left(\mathbb{R}^{+}\right)$,

hence $k(\infty):=\lim _{t \rightarrow \infty} k(t)=0$. These assumptions imply the the material enjoys the fading memory property, namely,

$$
\begin{aligned}
& \forall \varepsilon>0 \exists \tilde{a}=a\left(\varepsilon, \overline{\mathbf{g}}^{t}\right) \in \mathbb{R}^{+} \text {s.t. } \forall a>\tilde{a} \\
& \Rightarrow\left|\int_{0}^{\infty} \dot{k}(s+a) \overline{\mathbf{g}}^{t}(s) d s\right|<\varepsilon,
\end{aligned}
$$

which can be physically interpreted recalling that there is no heat flux when, at infinity, the thermal equilibrium is reached. The thermodynamical state of the conductor is characterized when, according to $[64,65$, 67], the thermodynamic state function $\sigma: \mathbb{R} \rightarrow \mathbb{R} \times$ $\mathbb{R}^{3}$ which associates $t \longmapsto \sigma(t) \equiv\left(u(t), \overline{\mathbf{g}}^{t}\right)$ is given. Hence, the following vectorial space 


$$
\begin{aligned}
\Gamma:= & \left\{\overline{\mathbf{g}}^{t}:(0, \infty) \rightarrow \mathbb{R}^{3}:\left|\int_{0}^{\infty} \dot{k}(s+\tau) \overline{\mathbf{g}}^{t}(s) d s\right|\right. \\
& <\infty, \forall \tau \geq 0\},
\end{aligned}
$$

is introduced to characterize physically admissible thermodynamical phenomena, namely those ones associated to a finite heat flux. Note, that the condition which defines the function space $\Gamma$, can be written also in a different form since, corresponding to any arbitrary $\varepsilon>0$, there exists a positive constant $\tilde{a}=$ $a\left(\varepsilon, \overline{\mathbf{g}}^{t}\right)$ s.t.

$$
\begin{aligned}
& \left|\int_{0}^{\infty} \dot{k}(s+a) \overline{\mathbf{g}}^{t}(s) d s\right| \\
& \quad=\left|\int_{0}^{\infty} k(s+a) \mathbf{g}(t-s) d s\right|<\varepsilon, \quad \forall a>\tilde{a} .
\end{aligned}
$$

Remarkably, the introduction of the integrated history of the temperature-gradient is crucial to write the fading memory condition under a form which is analogous to the fading memory condition in the case of isothermal viscoelasticity, as shown in the next subSection, the state of the heat conductor is characterized when, according to [64, 65, 67], the thermodynamic state function $\sigma: \mathbb{R} \rightarrow \mathbb{R} \times \mathbb{R}^{3}$ which associates $t \longmapsto$ $\sigma(t) \equiv\left(\theta(t), \overline{\mathbf{g}}^{t}\right)$ is given.

\subsection{Isothermal viscoelastic body with memory}

The aim of this sub-Section is to briefly summarize the analytical description of the model of an isothermal viscoelastic material with memory. Indeed, the historical as well as phenomenological ideas which have been developed throughout the literature are far beyond the present study. An overview concerning isothermal viscoelasticity is comprised in [68] and in [69] as well as in references therein. Gentili [68] studies minimum free energy and its connection to maximum recoverable work, while Deseri et al. [69], are concerned about free energies, again in isothermal viscoelasticity with the aim to treat applications to partial differential equations.

Here, first of all, a brief description of the essential properties which characterize the model of viscoelastic body [2, 41], are recalled. Denoted as $\Omega \subset \mathbb{R}^{3}$, the body configuration, the key assumptions on the viscoelastic material are the following ones [2, 41]:

- $\Omega$ is a connected set with a smooth boundary;
- $\Omega$ changes its shape according to linear viscoelasticity; that is, its deformation depends on time via present and past times, i.e., the deformation history of the material;

- The environment is assumed not to be affected by the presence of the body itself;

- no space dependence, i.e., $\mathbf{x}$-dependence is omitted under the assumption that the material satisfies both the isotropy and homogeneity conditions.

Hence, $[68,69]$ the quantities which are needed to describe this model are the strain tensor $\mathbf{E}(t)$, the stress tensor $\mathbf{T}(t)$, the relaxation modulus $\mathbb{G}(t)$ and its initial value, termed initial relaxation modulus $\mathbb{G}(0)$, where $t$ denotes the present time variable. The constitutive equation which characterizes a linear viscoelastic material is the classical Boltzmann-Volterra equation which relates the stress tensor $\mathbf{T}(t) \in S y m$ to the strain history tensor $E:(-\infty, t] \rightarrow$ Sym :

$$
\begin{aligned}
& \mathbf{T}(t)=\int_{0}^{\infty} \mathbb{G}(\tau) \dot{\mathbf{E}}(t-\tau) d \tau \quad \text { or } \\
& \mathbf{T}(t)=\mathbb{G}_{0} E(t)+\int_{0}^{\infty} \dot{\mathbb{G}}(\tau) \mathbf{E}(t-\tau) d \tau,
\end{aligned}
$$

where the fourth order tensor $\mathbb{G}(t)$ denotes the elastic modulus, $\mathbf{E}(t)$ the value of the strain at the time $t$ and $\mathbf{E}^{t}$ the past history defined by

$$
\begin{aligned}
& \mathbf{E}^{t}:(0, \infty) \rightarrow \text { Sym } \\
& t \longmapsto \mathbf{E}^{t}(s):=\mathbf{E}(t-s) .
\end{aligned}
$$

The elastic modulus is assumed such that its time derivative $\dot{\mathbb{G}} \in L^{1}\left(\mathbb{R}^{+}, \operatorname{Lin}(\operatorname{Sym})\right)$ so that, for all positive $t$,

$$
\mathbb{G}(t)=\mathbb{G}_{0}+\int_{0}^{t} \dot{\mathbb{G}}(s) d s, \quad \mathbb{G}_{0}:=\mathbb{G}(0),
$$

where the initial value of the elastic modulus $\mathbb{G}_{0}$ is termed instantaneous elastic modulus [68]; furthermore, since $\dot{\mathbb{G}} \in L^{1}$, then

$$
\mathbb{G}(\infty):=\lim _{t \rightarrow \infty} \mathbb{G}(t) \in \operatorname{Lin}(\operatorname{Sym})
$$

which represents the equilibrium elastic modulus. The state and the strain history of a viscoelastic body is characterized, according to [2, 68, 69], by a viscoelastic state function $\sigma: \mathbb{R} \rightarrow S y m \times S y m$, which associates $t \mapsto \sigma(t): \equiv\left(\mathbf{E}(t), \mathbf{E}^{t}\right)$. Hence, the viscoelastic state function is known when the strain tensor $\mathbf{E}(t)$ and the strain past history, $\mathbf{E}^{t}$ which belong to a suitable Hilbert space, are assigned. Physically 
meaningful viscoelastic phenomena, are characterized by a finite stress tensor $\mathbf{T}(t)$ for all times $t$ and hence they belong to the vectorial space

$$
\begin{aligned}
\Gamma & :=\left\{\mathbf{E}^{t}:(0, \infty) \rightarrow \text { Sym: }\left|\int_{0}^{\infty} \dot{\mathbb{G}}(s+\tau) \mathbf{E}^{t}(s) d s\right|\right. \\
& <\infty, \forall \tau \geq 0\} .
\end{aligned}
$$

According to [68], the material is said to enjoy the fading memory property when, corresponding to any arbitrary $\varepsilon>0$ there exists a positive constant $\tilde{a}=$ $a\left(\varepsilon, \mathbf{E}^{t}\right)$ s.t.

$\left|\int_{0}^{\infty} \dot{\mathbb{G}}(s+a) \mathbf{E}(t-s) d s\right|$

$=\left|\int_{0}^{\infty} \mathbb{G}(s+a) \dot{\mathbf{E}}(t-s) d s\right|<\varepsilon, \quad \forall a>\tilde{a}$.

The same way of reasoning followed in the previous sub-Section allows to establish that admissible states are only those ones related to a finite viscoelastic work, and the equivalence between any couple of different states as those ones associated to the same value of the viscoelastic work. Note the analogy between the two conditions (14) and (8): it shows that the couples, $\mathbb{G}, \mathbf{E}^{t}$, on one side, and $k, \overline{\mathbf{g}}^{t}$, on the other one, play, respectively, the same role in the two different formulae.

Remark Note that definition (14), and also (8), is not affected by the generalization here considered since when $\mathbb{G}(t)$, and also $k(t)$, is allowed to admit an infinite limit $t \rightarrow 0, \dot{\mathbb{G}}$, and also $\dot{k}$, is not integrable at the origin; however, it belongs to $L^{1}(a,+\infty), \forall a>0$.

\section{Heat conduction evolution problem}

In this Section the evolution problem of a rigid heat conductor with memory is considered. The classical regular initial boundary value problem with homogeneous initial and Neumann boundary conditions is recalled. In the next sub-Section the corresponding singular problem is studied.

\subsection{Regular memory kernel}

The one-dimensional evolution equation which models rigid heat conduction with memory can be written $u_{t}=\int_{0}^{t} k(\tau) u_{x x}(x, t-\tau) d \tau+f(x, t)$,

where in the term $f$, which represents the source term, also the past history of the material is incorporated. Generally, $f$ is assumed to be sufficiently regular to allow integration and partial derivation with respect to time and, in addition, is also supposed to be $L^{2}$ integrable in the space variable. This choice allows to write the corresponding evolution problem, when initial and boundary conditions are assigned, under the form of a Volterra equation. Here homogeneous Neumann boundary conditions

$u_{x}(0, t)=u_{x}(L, t)=0, \quad \forall t>0$,

and the initial condition, at $t=0$ are imposed. The three-dimensional initial boundary value problem with homogeneous initial and Dirichlet boundary conditions is studied in [62]. Equation (15), on introduction of $s:=t-\tau$, reads

$u_{t}=\int_{0}^{t} k(t-s) u_{x x}(x, s) d s+f(x, t)$.

Derivation with respect to time of the latter gives

$u_{t t}=k(0) u_{x x}+\int_{0}^{t} \dot{k}(t-\tau) u_{x x}(\tau) d \tau+f_{t}$,

then, consider the i.b.v.p. obtained imposing on (18) the following initial and boundary conditions

$\left.u\right|_{t=0}=u_{0}(x),\left.\quad u_{t}\right|_{t=0}=f(x, 0)$,

$\left.u_{x}\right|_{\partial \Omega \times(0, T)}=0, \quad t<T$,

where $\Omega$ denotes the interval $(0, L), L>0$. Note that the linear problem (18)-(19) is of the same form of the integro-differential problem which models the viscoelastic body evolution (26)-(27), in the next Section, hence, when the heat flux relaxation function $k$, finite at the origin, satisfies the thermodynamical assumptions (5), then Dafermos' results [29, 30] imply the existence and uniqueness of the solution.

\subsection{Singular memory kernel}

In this sub-Section, the one-dimensional singular heat conduction problem is studied. Now, aiming to model a wider class of materials with memory, the functional 
requirements on the heat flux relaxation function $k$ are relaxed, removing the condition $\dot{k} \in L^{1}(0, T)$, thus

$$
k(t)>0, \quad \dot{k}(t) \leq 0, \quad \ddot{k}(t) \geq 0, \quad t \in(0, \infty),
$$

and

$$
k \in L^{1}(0, T) \cap C^{2}(0, T) \quad \forall T \in \mathbb{R}^{+} .
$$

Now, the same method valid in studying the viscoelasticity problem, can be applied straightforwardly. Hence, key steps of the Approximation Strategy can be sketched as follows:

- construct suitable regular approximated problems;

- find approximated solutions $u^{\varepsilon}, 0<\varepsilon \ll 1$;

- show the existence of $u:=\lim _{\varepsilon \rightarrow 0} u^{\varepsilon}$;

- prove the uniqueness of the limit solution $u$ which represents a weak solution admitted by the singular problem.

Accordingly, first of all the approximated problems are introduced: let $k^{\varepsilon}(\cdot):=k(\varepsilon+\cdot)$ then, the problem $P_{D, \text { heat }}^{\varepsilon}$ can be defined

$$
P_{D, h e a t}^{\varepsilon}: u_{t t}^{\varepsilon}=k^{\varepsilon}(0) u_{x x}^{\varepsilon}+\int_{0}^{t} \dot{k}^{\varepsilon}(t-\tau) u_{x x}^{\varepsilon}(\tau) d \tau+f \text {. }
$$

The integro-differential problem (22), when conditions (19) are imposed on $u^{\varepsilon}$, is regular since $k^{\varepsilon}(0)$ is finite and, therefore, $[29,30]$ admits a unique solution. It, in addition, corresponding to each value of $\varepsilon$, is equivalent, to the following integral problem

$$
P_{I, h e a t}^{\varepsilon}: u^{\varepsilon}(t)=\int_{0}^{t} K^{\varepsilon}(t-\tau) u_{x x}^{\varepsilon}(\tau) d \tau+u_{0}+\int_{0}^{t} f(\tau) d \tau,
$$

where,

$$
K(\xi):=\int_{0}^{\xi} k(\tau) d \tau \quad K(0)=0,
$$

is well defined since $k \in L^{1}(0),, \forall T \in \mathbb{R}^{+} . K$ is termed integrated relaxation function. Partial derivation w.r.to $t$, twice, of (23) delivers (22) together with conditions (19). Note that, on use of (24), when $\varepsilon=0$ and the superscripts ${ }^{0}$ are omitted, the following well defined integral problem

$$
P_{I, \text { heat }}: u(t)=\int_{0}^{t} K(t-\tau) u_{x x}(\tau) d \tau+u_{0}+\int_{0}^{t} f(\tau) d \tau,
$$

is obtained.

\section{Viscoelastic problem}

In this Section the linear integro-differential problem in the case of the one-dimensional viscoelastic classical model, is considered. Thus, from here on, the tensor $\mathbb{G}$ is denoted as $G$ since we are restricting our attention to the one-dimensional case. In the starting sub-Section, following the same lines as in the heat conduction problem, the classical regular case is recalled to point out the functional requirements the kernel satisfies in such a case. Then, in the subsequent sub-Section, the regularity requirements on $G$ are relaxed.

\subsection{Regular memory kernel}

According to Dafermos [29, 30], such a model can be represented by

$$
\begin{aligned}
& u_{t t}=G(0) u_{x x}+\int_{0}^{t} \dot{G}(t-\tau) u_{x x}(\tau) d \tau+f, \\
& u(\cdot, 0)=u_{0}, \quad u_{t}(\cdot, 0)=u_{1} \text { in } \Omega ; \quad u_{x}=0 \\
& \text { on } \Sigma=\partial \Omega \times(0, T),
\end{aligned}
$$

where $\Omega=(0, L)$, the initial and boundary conditions are assigned while, respectively, $u$ and $f$ denote the displacement and the external force which includes also the history of the material. In addition, according to the model assumptions (see, for instance [2, 41])

$$
G(t)>0, \quad \dot{G}(t) \leq 0, \quad \ddot{G}(t) \geq 0, \quad t \in(0, \infty)
$$

and, in addition,

$$
\begin{aligned}
\dot{G} \in L^{1}\left(\mathbb{R}^{+}\right) \quad G(t) & =G_{0} \\
+\int_{0}^{t} \dot{G}(s) d s \quad G_{\infty} & :=\lim _{t \rightarrow \infty} G(t) ;
\end{aligned}
$$


$G$ enjoys the fading memory property (14). When the relaxation modulus satisfies assumptions (29), the problem (26)-(27), here termed Regular Memory Kernel, admits a unique solution according to Dafermos [29, 30].

\subsection{Singular memory kernel}

In many applications, however, to model the physical behavior of new materials or polymers, the relaxation modulus does not satisfy the functional requirements (29). Hence, to model a wider class of materials with memory, the functional requirements imposed on $G$ are relaxed, that is $G$ is assumed to satisfy (28) further to the condition

$$
G \in L^{1}(0, T) \cap C^{2}(0, T) \quad \forall T \in \mathbb{R} .
$$

Note that, now, according to (28) and (30), the relaxation function $G(t)$ is not required to be finite at $t=0$, since $\lim _{t \rightarrow 0^{+}} G(t)=+\infty$, then Eq. (26) needs to be replaced by a different one. Here the method, devised in [61], is adapted to the case of Neumann boundary conditions. The three-dimensional generalization is under investigation [63]. The key steps of the Approximation Strategy can be sketched as follows:

- construct suitable regular approximated problems;

- find approximated solutions $u^{\varepsilon}, 0<\varepsilon \ll 1$;

- show the existence of $u:=\lim _{\varepsilon \rightarrow 0} u^{\varepsilon}$;

- prove the uniqueness of the limit solution $u$ which represents a weak solution admitted by the singular problem.

Accordingly, first of all, ad hoc regular kernel problems termed approximated problems are introduced. Following the same strategy already shown in the previous Section referring to linear rigid heat conduction with memory, let $G^{\varepsilon}(\cdot):=G(\varepsilon+\cdot)$ then, the integro-differential problem $P_{D, v i s c o}^{\varepsilon}$ can be defined

$$
P_{D, v i s c o}^{\varepsilon}: u_{t t}^{\varepsilon}=G^{\varepsilon}(0) u_{x x}^{\varepsilon}+\int_{0}^{t} \dot{G}^{\varepsilon}(t-\tau) u_{x x}^{\varepsilon}(\tau) d \tau+f,
$$

together with the initial and boundary conditions

$$
\begin{gathered}
\left.u^{\varepsilon}\right|_{t=0}=u_{0}(x),\left.\quad u_{t}^{\varepsilon}\right|_{t=0}=u_{1}(x),\left.\quad u_{x}^{\varepsilon}\right|_{\partial \Omega \times(0, T)}=0, \\
t<T .
\end{gathered}
$$

The latter is a regular problem since $G^{\varepsilon}(0)$ is finite and, therefore, the initial boundary value problem (31)-
(32) admits a unique solution. In addition, corresponding to each value of $\varepsilon$, it is equivalent to the following integral equation

$$
\begin{aligned}
P_{I, v i s c o}^{\varepsilon}: u^{\varepsilon}(t)= & \int_{0}^{t} K^{\varepsilon}(t-\tau) u_{x x}^{\varepsilon}(\tau) d \tau+u_{1 t}+u_{0} \\
& +\int_{0}^{t} d \tau \int_{0}^{\tau} f(\xi) d \xi
\end{aligned}
$$

where

$$
K(\xi):=\int_{0}^{\xi} G(\tau) d \tau \quad K(0)=0,
$$

is well defined since $G \in L^{1}(0, T), \forall T \in \mathbb{R}^{+} . K$ is termed integrated relaxation function. Partial derivation w.r.to $t$, twice, of (33) delivers (31) together with initial and boundary conditions (32).

Again, as observed in the case of the integral equation (25), when $\varepsilon=0$ and the superscripts ${ }^{0}$ are omitted, also

$$
\begin{aligned}
P_{I, v i s c o}: u(t)= & \int_{0}^{t} K(t-\tau) u_{x x}(\tau) d \tau+u_{1} t+u_{0} \\
& +\int_{0}^{t} d \tau \int_{0}^{\tau} f(\xi) d \xi
\end{aligned}
$$

is well defined.

Notably, even if the physical meaning of the involved quantities is different in the two cases, the integral equations (23) and (33) share the same terms which depend on $\varepsilon$ : the other ones, related to the different initial and boundary conditions, do not depend on $\varepsilon$ and, hence, are unchanged when the limit $\varepsilon \rightarrow 0$ is performed.

\section{Existence and uniqueness of the limit solution}

This Section is devoted to the existence and uniqueness of the limit solution in both the cases of onedimensional singular problems considered. Indeed, following the same method, both (23) and (33) can be proved to admit and unique solution. To improve readability, in this Section only the crucial steps to prove the results are given while details on proofs are postponed to the Appendix.

Here a unified approach to both the problems is given. Accordingly, the following existence Theorem can be stated referring to both the integral problems $P_{I, h e a t}^{\varepsilon}$ as well as $P_{I, v i s c o}^{\varepsilon}$. 
Remark The existence and uniqueness results are proved via the same method in both the different problems (23) and (33) since they share the same integral term which depends on $\varepsilon$, namely

$$
\int_{0}^{t} K^{\varepsilon}(t-\tau) u_{x x}^{\varepsilon}(\tau) d \tau
$$

Theorem 1 Given $u^{\varepsilon}$ solution to the integral problem $P_{I, \text { heat }}^{\varepsilon}$ in (23), or $P_{I, \text { visco }}^{\varepsilon}$ (33), then

$$
\exists u(t)=\lim _{\varepsilon \rightarrow 0} u^{\varepsilon}(t) \quad \text { in } \quad L^{2}(Q), \quad Q=\Omega \times(0, T) .
$$

\section{Proof's Outline}

- weak formulation, on introduction of test functions $\varphi \in H^{1}(\Omega \times(0, T))$ s.t. $\varphi_{x}=0$ on $\partial \Omega$;

- consider separately the terms without $\varepsilon$;

- the terms with $u^{\varepsilon}$ and $K^{\varepsilon}$;

- prove convergence via Lebesque's Theorem.

Furthermore, the following uniqueness Theorems, respectively, concerning the heat and viscoelastic problems can be proved.

Theorem 2 The integral problems (25) as well as (35) admit a unique weak solution.

Proof's Outline in both cases, the result is proved by contradiction assuming there are two different solution and, then, showing that such an assumption leads to a contradiction. Note that the proof is unified since, on use of linearity, given two different solutions $v$ and $\tilde{v}$, of any of the two Eqs. (25) or (35), then their difference is again a solution. In addition, let $w:=$ $v-\tilde{v}$, it follows to satisfy

$w(t)=\int_{0}^{t} K(t-\tau) w_{x x}(\tau) d \tau$,

in both the considered problems. ${ }^{1}$

\section{Conclusions and perspectives}

A crucial role in achieving most of the results presented [59, 60], is played by the free energy. Indeed, in the case of the singular problems both in

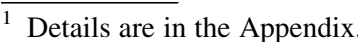

viscoelasticity and in rigid thermodynamics with memory $[61,62]$ are based on the free energy in one dimensional as well as in the general three-dimensional case [63]. This, aspect is one of the crucial ones which are currently under investigation, in particular, aiming to study evolution problems in the case of viscoelastic fluids as modeled in [11]. Also the a priori estimates both, in the one-dimensional as well as in higher dimensions magneto-viscoelasticity problems $[59,60]$, are based on the free energy. The perspective research aims to extend the study of singular kernel problems to further materials whose evolution can be modeled in a similar way. Already under investigation is the case of a magneto-viscoelasticity problem, when the singular viscoelastic behaviour is coupled with the magnetic effects [70].

Acknowledgments The partial financial support of G.N.F.M.I.N.d.A.M. and of SAPIENZA Università di Roma are gratefully acknowledged.

\section{Appendix}

This Appendix comprises the proofs of both Theorems in Sect. 5. They refer to the rigid heat conduction problem. Indeed, the difference is mainly in the physical meaning of the quantities involved.

To prove Theorem 1, the following Lemma provides a needed estimate. ${ }^{2}$

Lemma 1 Let $u^{\varepsilon}$ be the unique solution to the problem (18)-(19), then

$\frac{1}{2} \int_{\Omega}\left|u_{x}\right|^{2} d x+\frac{1}{2} \int_{\Omega}\left|u_{t}\right|^{2} d x \leq \gamma e^{T} C\left(f, u_{0}\right)$,

where $\gamma=\max \left\{(k(T+1))^{-1}, 1\right\}$.

Proof The statement follows when (18) is multiplied by $u_{t}$, then, integrated over $\Omega$, after use of various integrations by parts, also integrating over the time interval $(0, t)$. Key tools are represented by the thermodynamical assumptions (20) on the heat flux relaxation function and by taking into account the assigned boundary conditions, Specifically, (18) multiplied by $u_{t}$ and integrated over $\Omega$, when all the

\footnotetext{
${ }^{2}$ An analogous result can be proved in the viscoelasticity problem. The Dirichlet boundary value problem with assigned initial conditions is in [61] here the Neumann problem is considered.
} 
superscripts $\varepsilon$ are removed to simplify the notation, gives:

$$
\begin{aligned}
& \frac{1}{2} \frac{d}{d t} \int_{\Omega}\left|u_{t}\right|^{2} d x+\int_{\Omega} k(t+\varepsilon) u_{x} u_{x t} d x \\
& \quad+\int_{\Omega} u_{t}(t) d x \int_{0}^{t} \dot{k}(s+\varepsilon)\left[u_{x x}(t)-u_{x x}(t-s)\right] d s \\
& =\int_{\Omega} f_{t} u_{t} d x
\end{aligned}
$$

which can be written as

$$
\begin{aligned}
& \frac{1}{2} \frac{d}{d t} \int_{\Omega}\left|u_{t}\right|^{2} d x+\frac{1}{2} \frac{d}{d t} \int_{\Omega} k(t+\varepsilon)\left|u_{x}\right|^{2} d x \\
& \quad-\frac{1}{2} \int_{\Omega} \dot{k}(t+\varepsilon)\left|u_{x}\right|^{2} d x \\
& \quad-\int_{\Omega} d x \int_{0}^{t} \dot{k}(s+\varepsilon) u_{x t}\left[u_{x}(t)-u_{x}(t-s)\right] d s \\
& =\int_{\Omega} f_{t} u_{t} d x .
\end{aligned}
$$

In particular, the last term can be written

$$
\begin{aligned}
& -\int_{\Omega} \int_{0}^{t} \dot{k}(s+\varepsilon) u_{x t}\left[u_{x}(t)-u_{x}(t-s)\right] d x d s \\
= & -\frac{1}{2} \frac{d}{d t} \int_{0}^{t} d s \int_{\Omega} \dot{k}(s+\varepsilon)\left|u_{x}(t)-u_{x}(t-s)\right|^{2} d x \\
& +\frac{1}{2} \int_{\Omega} \dot{k}(t+\varepsilon)\left|u_{x}(t)-u_{x}(0)\right|^{2} d x \\
& -\int_{\Omega} \int_{0}^{t} \dot{k}(s+\varepsilon) u_{x t}(t-s)\left[u_{x}(t)-u_{x}(t-s)\right] d x d s,
\end{aligned}
$$

which, since

$$
2 u_{x t}(t-s)\left[u_{x}(t)-u_{x}(t-s)\right]=\frac{d}{d s}\left|u_{x}(t)-u_{x}(t-s)\right|^{2},
$$

can be further manipulated to deliver

$$
\begin{aligned}
& -\int_{\Omega} \int_{0}^{t} \dot{k}(s+\varepsilon) u_{x t}\left[u_{x}(t)-u_{x}(t-s)\right] d x d s \\
= & -\frac{1}{2} \frac{d}{d t} \int_{0}^{t} d s \int_{\Omega} \dot{k}(s+\varepsilon)\left|u_{x}(t)-u_{x}(t-s)\right|^{2} d x \\
& +\frac{1}{2} \int_{\Omega} \int_{0}^{t} \ddot{k}(s+\varepsilon)\left|u_{x}(t)-u_{x}(t-s)\right|^{2} d x d s,
\end{aligned}
$$

which, substituted in (40) implies

$$
\begin{aligned}
\frac{1}{2} & \frac{d}{d t} \int_{\Omega} k(t+\varepsilon)\left|u_{x}\right|^{2} d x-\frac{1}{2} \frac{d}{d t} \int_{0}^{t} d s \int_{\Omega} \dot{k}(s+\varepsilon) \mid u_{x}(t) \\
& -\left.u_{x}(t-s)\right|^{2} d x+\frac{1}{2} \frac{d}{d t} \int_{\Omega}\left|u_{t}\right|^{2} d x \\
= & \int_{\Omega} f_{t} u_{t} d x+\frac{1}{2} \int_{\Omega} \dot{k}(t+\varepsilon)\left|u_{x}\right|^{2} d x \\
& -\frac{1}{2} \int_{0}^{t} d s \int_{\Omega} \ddot{k}(s+\varepsilon)\left|u_{x}(t)-u_{x}(t-s)\right|^{2} d x .
\end{aligned}
$$

The latter, integrated over the time interval $(0, t)$, taking into account the conditions (20), delivers

$$
\begin{aligned}
& \frac{1}{2} \int_{\Omega} k(t+\varepsilon)\left|u_{x}\right|^{2} d x+\frac{1}{2} \int_{\Omega}\left|u_{t}\right|^{2} d x \\
& \quad \leq \int_{\Omega} \int_{0}^{t} f_{t} u_{t} d x d s+\frac{1}{2} \int_{\Omega} k(\varepsilon)|u(0)|^{2} d x \\
& \quad+\frac{1}{2} \int_{\Omega}|f(x, 0)|^{2} d x ;
\end{aligned}
$$

which allows to write

$$
\begin{aligned}
& \frac{1}{2} \int_{\Omega} k(t+\varepsilon)\left|u_{x}\right|^{2} d x+\frac{1}{2} \int_{\Omega}\left|u_{t}\right|^{2} d x \\
& -\int_{0}^{t} \int_{\Omega}\left|u_{t}\right|^{2} d x d s \leq C\left(f, u_{0}\right),
\end{aligned}
$$

and, Gronwall's Lemma implies

$\frac{1}{2} \int_{\Omega} k(t+\varepsilon)\left|u_{x}\right|^{2} d x+\frac{1}{2} \int_{\Omega}\left|u_{t}\right|^{2} d x \leq e^{T} C\left(f, u_{0}\right)$.

Since $k(t+\varepsilon) \geq k(T+1)$,

$\frac{1}{2} \int_{\Omega}\left|u_{x}\right|^{2} d x+\frac{1}{2} \int_{\Omega}\left|u_{t}\right|^{2} d x \leq \gamma e^{T} C\left(f, u_{0}\right)$,

wherein $\gamma=\max \left\{(k(T+1))^{-1}, 1\right\}$.

Proof of Theorem 1 The existence of the solution admitted by the singular problem is given by $u=\lim _{\varepsilon_{h} \rightarrow 0} u^{\varepsilon_{h}}$, where $u^{\varepsilon_{h}}$ is a solution of (18)-(19) i.e., it solves

$$
\begin{aligned}
P^{\varepsilon_{h}} & : u^{\varepsilon_{h}}(t)=\int_{0}^{t} K^{\varepsilon_{h}}(t-\tau) u_{x x}^{\varepsilon_{h}}(\tau) d \tau \\
+ & \int_{0}^{t} f(\tau) d \tau, \quad K^{\varepsilon_{h}}(\xi):=\int_{0}^{\xi} k\left(\varepsilon_{h}+\tau\right) d \tau .
\end{aligned}
$$


This thesis is proved following the outline given in Sect. 5: here each step is given.

- weak formulation of the integral problem;

consider the test functions $\varphi$, which depend on both time and space variables, subject to assigned i.c. and homogeneous Neumann boundary conditions on the boundary, $\partial \Omega$, of $\Omega=(0, L) \subset \mathbb{R}$,

$$
\begin{gathered}
\varphi \in C^{\infty}(Q), \quad Q=\Omega \times(0, T), \\
\text { s.t. }\left.\quad \varphi_{x}\right|_{\partial \Omega}=0 \quad \forall t \in(0, T) .
\end{gathered}
$$

Multiplication of (48) by $\varphi$ and integration over $Q$ gives

$$
\begin{aligned}
& \iint_{Q} u^{\varepsilon_{h}}(t) \varphi d x d t=\iint_{Q} \varphi \\
& \left\{\int_{0}^{t} K^{\varepsilon_{h}}(t-\tau) u_{x x}^{\varepsilon_{h}}(\tau) d \tau+\int_{0}^{t} f(\tau) d \tau\right\} d x d t .
\end{aligned}
$$

- consider separately the terms without $\varepsilon$;

The term

$$
\iint_{Q} \varphi\left\{\int_{0}^{t} f(\tau) d \tau\right\} d x d t
$$

does not depend on $\varepsilon_{h}$ and, hence, it is unchanged in the limit $\varepsilon_{h} \rightarrow 0$. Furthermore, it is bounded since the history $f$ of the material with memory is assumed to be regular and $Q$ is bounded too.

- consider the term with $u^{\varepsilon}$ and $K^{\varepsilon}$;

the only term which needs to be considered is

$$
\int_{0}^{t} K^{\varepsilon_{h}}(t-\tau) u^{\varepsilon_{h}}(\tau) d \tau=\int_{0}^{t} K^{\varepsilon_{h}}(s) u^{\varepsilon_{h}}(t-s) d s
$$

since it depends on $\varepsilon$. Both the test functions $\varphi$ as well as $u^{\varepsilon_{h}}$ satisfy the homogeneous Neumann b.c. (49), then,

$$
\begin{aligned}
& \iint_{Q} \varphi d x d t \int_{0}^{t} K^{\varepsilon_{h}}(s) u_{x x}^{\varepsilon_{h}}(t-s) d s \\
& =\iint_{Q} \varphi_{x x} d x d t \int_{0}^{t} K^{\varepsilon_{h}}(s) u^{\varepsilon_{h}}(t-s) d s .
\end{aligned}
$$

Now, adding and subtracting $\iint_{Q} \varphi_{x x} d x d t$ $\int_{0}^{t} K(s) u^{\varepsilon_{h}}(t-s) d s$, the latter gives

$$
\begin{aligned}
& \iint_{Q} \varphi_{x x} \int_{0}^{t} K^{\varepsilon_{h}}(t-\tau) u^{\varepsilon_{h}}(\tau) d \tau d x d t \\
& =\iint_{Q} \varphi_{x x} \int_{0}^{t}\left[K^{\varepsilon_{h}}(s)-K(s)\right] u^{\varepsilon_{h}}(t-s) d s d x d t \\
& \quad+\iint_{Q} \varphi_{x x} \int_{0}^{t} K(s) u^{\varepsilon_{h}}(t-s) d s d x d t
\end{aligned}
$$

Observe that

Lemma 2 Given the integral problem (48)-(49), then

$\lim _{\varepsilon_{h} \rightarrow 0} \iint_{Q} \varphi_{x x} \int_{0}^{t}\left[K^{\varepsilon_{h}}(s)-K(s)\right] u^{\varepsilon_{h}}(t-s) d s d x d t=0$.

Proof of Lemma $\forall(x, t) \in Q=\Omega \times(0, T) \Longrightarrow$ $|u| \leq C|\Omega|$, and $\left|\varphi_{x x}\right| \leq M$, furthermore

$$
\left|K^{\varepsilon_{h}}(s)-K(s)\right|=\left|K\left(\varepsilon_{h}+s\right)-K(s)\right|=\int_{s}^{\varepsilon_{h}+s} k(\tau) d \tau,
$$

hence, since $k \in L^{1}(0, T)$, Lebesgue's Theorem implies the limit convergence (55) and the Lemma is proved.

Hence, recalling also estimate (38), the Theorem is proved that is

(a) $u^{\varepsilon_{h}} \longrightarrow u$ weakly in $H^{1}\left(0, T, H^{1}(\Omega)\right)$ as $\varepsilon_{h} \rightarrow 0$;

(b) $u^{\varepsilon_{h}} \longrightarrow u$ strongly in $L^{2}(D)$ as $\varepsilon_{h} \rightarrow 0$.

Proof of Theorem 2 (uniqueness of the solution admitted by (25) and by (35))

The thesis is proved by contradiction; thus, assume $v$ and $\tilde{v}, v \neq \tilde{v}$, both satisfy the linear equation (25). Then, also $w:=v-\tilde{v}$, as any linear combination of $v$ and $\tilde{v}$, is a solution of the same equation. Let $w:=$ $v-\tilde{v}$, it is a solution to

$w(t)=\int_{0}^{t} K(t-\tau) w_{x x}(\tau) d \tau$,

subject to the assigned homogeneous initial and boundary Neumann conditions. Accordingly, let $\Omega=$ $(0, \pi)$, i.e., for convenience, let $L=\pi, w_{x}(0, t)=$ $w_{x}(\pi, t)=0, \forall t \in(0, T)$. The test functions $\psi(x, t)$ can be chosen as 
$\psi_{m}(x, t)=\varphi(t) \cos (m x), \quad m \in \mathbb{N}$

which satisfy the assigned initial and boundary conditions, then, the solution $w(x, t)$ can be written

$w(x, t)=\sum_{n=1}^{\infty} \alpha_{n}(t) \cos (n x)$.

The weak solution, when $Q=(0, \pi) \times(0, T)$ and $\psi(x, t)$ denotes any test function, reads

$$
\begin{aligned}
& \iint_{Q} w(t) \psi(x, t) d x d t=\iint_{Q} \psi(x, t) \\
& \int_{0}^{t} K(t-\tau) w_{x x}(\tau) d \tau d x d t
\end{aligned}
$$

or equivalently

$$
\begin{aligned}
& \iint_{Q} w(t) \psi(x, t) d x d t=\iint_{Q} \psi_{x x}(x, t) \\
& \int_{0}^{t} K(t-\tau) w(\tau) d \tau d x d t .
\end{aligned}
$$

Substitution of the expressions of $w$ and $\psi$, in turn, (59) and (58), combined with the orthogonality of the cosine functions, gives

$$
\int_{0}^{T} \varphi(t)\left[\alpha_{m}(t)-\int_{0}^{t} K(t-\tau) m^{2} \alpha_{m}(t) d \tau\right] d t=0 .
$$

Then, since the test function $\varphi$ are arbitrary, it follows:

$$
\alpha_{m}(t)=\int_{0}^{t} K(t-\tau) m^{2} \alpha_{m}(t) d \tau
$$

which implies

$$
\left|\alpha_{m}(t)\right| \leq K(T) m^{2} \int_{0}^{t}\left|\alpha_{m}(\tau)\right| d \tau,
$$

and, via Gronwall's Lemma, $\alpha_{m}(t)=0 \forall m \in \mathbb{N}$.

\section{References}

1. Rabotnov YuN (1980) Elements of hereditary solid mechanics. Mir Publishers, Moscow

2. Fabrizio M, Morro A (1992) Mathematical problems in linear viscoelasticity. SIAM studies in applied mathematics, vol 12. Society for Industrial and Applied Mathematics (SIAM), Philadelphia. ISBN 0-89871-266-1

3. Tschoegl NW (1989) The phenomenological theory of linear viscoelastic behavior. An introduction. Springer, Berlin. ISBN 3-540-19173-9

4. Borcherdt RD (2009) Viscoelastic waves in layered media. Cambridge University Press, Cambridge

5. Mainardi F (2010) Fractional calculus and waves in linear viscoelasticity. An introduction to mathematical models. Imperial College Press, London

6. Miara B, Stavroulakis G, Valente V (eds) (2007) Topics on mathematics for smart systems. World Scientific Publishing Co., Pte. Ltd., Hackensack

7. Fabrizio M, Lazzari B, Morro A (eds, 2002) Mathematical models and methods for smart materials. Series on advances in mathematics for applied sciences, vol 62. World Scientific Publishing Co., Inc., River Edge

8. Fabrizio M (2014) Fractional rheological models for thermomechanical systems. Dissipation and free energies. Fract Calc Appl Anal 17(1):206-223

9. Deseri L, Zingales M, Pollaci P (2014) The state of fractional hereditary materials (FHM). Discret Contin Dyn Syst B 19(7):2065-2089. doi:10.3934/dcdsb.2014. 19.2065

10. Carillo S, Giorgi C, Grasselli M (2014) Special issue dedicated to Mauro Fabrizio's 70th birthday FOREWORD. Discret Contin Dyn Syst B 19(7):I-I Special Issue. doi:10. 3934/dcdsb.2014.19.7i

11. Amendola G, Carillo S, Golden JM, Manes A (2014) Viscoelastic fluids: free energies, differential problems and asymptotic behaviour. Discret Contin Dyn Syst B 19(7):1815-1835. doi:10.3934/dcdsb.2014.19.1815

12. Berti A, Giorgi C, Morro A (2014) Mathematical modeling of phase transition and separation in fluids: a unified approach. Discret Contin Dyn Syst B 19(7):1889-1909. doi:10.3934/dcdsb.2014.19.1889

13. Bonetti E, Colli P, Gilardi G (2014) Singular limit of an integrodifferential system related to the entropy balance. Discret Contin Dyn Syst B 19(7):1935-1953. doi:10.3934/ dcdsb.2014.19.1935

14. Cavalcanti MM, Domingos Cavalcanti VN, Lasiecka I, Falco Nascimento FA (2014) Intrinsic decay rate estimates for the wave equation with competing viscoelastic and frictional dissipative effects. Discret Contin Dyn Syst B 19(7):1987-2012. doi:10.3934/dcdsb.2014.19.1987

15. Chirită S (2014) Spatial behavior in the vibrating thermoviscoelastic porous materials. Discret Contin Dyn Syst B 19(7):2027-2038. doi:10.3934/dcdsb.2014.19.2027

16. Eleuteri M, Kopfov J, Krejčí P (2014) Fatigue accumulation in a thermo-visco-elastoplastic plate. Discret Contin Dyn Syst B 19(7):2091-2109. doi:10.3934/dcdsb.2014.19.2091

17. Franchi F, Lazzari B, Nibbi R (2014) Uniqueness and stability results for non-linear Johnson-Segalman viscoelasticity and related models. Discret Contin Dyn Syst B 19(7):2111-2132. doi:10.3934/dcdsb.2014.19.2111

18. Giorgi C, Grandi D, Pata V (2014) On the Green-Naghdi type III heat conduction model. Discret Contin Dyn Syst B 19(7):2133-2143. doi:10.3934/dcdsb.2014.19.2133

19. Giusteri GG, Marzocchi A, Musesti A (2014) Nonlinear free fall of one-dimensional rigid bodies in hyperviscous fluids. 
Discret Contin Dyn Syst B 19(7):2145-2157. doi:10.3934/ dcdsb.2014.19.2145

20. Al Horani M, Favini A (2014) Inverse problems for singular differential-operator equations with higher order polar singularities. Discret Contin Dyn Syst B 19(7):2159-2168. doi:10.3934/dcdsb.2014.19.2159

21. Ieşan D (2014) Strain gradient theory of porous solids with initial stresses and initial heat flux. Discret Contin Dyn Syst B 19(7):2169-2187. doi:10.3934/dcdsb.2014.19.2169

22. Jordan PM (2014) Second-sound phenomena in inviscid, thermally relaxing gases. Discret Contin Dyn Syst B 19(7):2189-2205. doi:10.3934/dcdsb.2014.19.2189

23. Roubiček T, Tomassetti G (2014) Thermomechanics of hydrogen storage in metallic hydrides: modeling and analysis. Discret Contin Dyn Syst B 19(7):2313-2333. doi:10. 3934/dcdsb.2014.19.2313

24. Svanadze M (2014) On the theory of viscoelasticity for materials with double porosity. Discret Contin Dyn Syst B 19(7):2335-2352. doi:10.3934/dcdsb.2014.19.2335

25. Andreucci D, Bellaveglia D, Cirillo ENM, Marconi S (2014) Effect of intracellular diffusion on current-voltage curves in potassium channels. Discret Contin Dyn Syst B 19(7):1837-1853. doi:10.3934/dcdsb.2014.19.1837

26. Bissell J, Straughan B (2014) Discontinuity waves as tipping points: applications to biological \& sociological systems. Discret Contin Dyn Syst B 19(7):1911-1934. doi:10. 3934/dcdsb.2014.19.1911

27. Cherfils L, Miranville A, Zelik S (2014) On a generalized Cahn-Hilliard equation with biological applications. Discret Contin Dyn Syst B 19(7):2013-2026. doi:10.3934/ dcdsb.2014.19.2013

28. De Angelis M, Renno P (2014) Asymptotic effects of boundary perturbations in excitable systems. Discret Contin Dyn Syst Ser B 19(7):2039-2045. doi:10.3934/dcdsb.2014. 19.2039

29. Dafermos CM (1970) An abstract Volterra equation with applications to linear viscoelasticity. J Differ Equ 7:554-569

30. Dafermos CM (1970) Asymptotic stability in viscoelasticity. Arch Rational Mech Anal 37:297-308

31. Boltzmann L (1876) Zur theorie der elastichen nachwirkung. Ann phys chem 77:624-654

32. Zimm BH (1956) Dynamics of polymer molecules in dilute solutions: viscoelasticity, flow birefringence and dielectric loss. J Chem Phys 24:269-278

33. Rouse PE (1953) A theory of the linear viscoelastic properties of dilute solutions of coiling polymers. J Chem Phys 21:1272-1280

34. Curtiss CF, Bird RB (1981) A kinetic theory for polymer melts, I. The equation for the single link orientational distribution function. J Chem Phys 74:2016-2025

35. Renardy M, Hrusa WJ, Nohel JA (1987) Mathematical problems in viscoelasticity. Longman Scientific and Technical, Wiley, New York

36. Adolfsson K, Enelund M, Olsson P (2005) On the fractional order model of viscoelasticity. Mech Time Depend Mater 9:15-34

37. Enelund M, Mähler L, Runesson K, Josefson BL (1999) Formulation and integration of the standard linear viscoelastic solid with fractional order rate laws. Int J Solids Struct 36:2417-2442
38. Enelund M, Olsson P (1999) Damping described by fading memory, Analysis and application to fractional derivative models. Int J Solids Struct 36:939-970

39. Desch W, Grimmer R (1989) Singular relaxation moduli and smoothing in three-dimensional viscoelasticity. Trans Am Math Soc 314:381-404

40. Desch W, Grimmer R (1989) Smoothing properties of linear Volterra integrodifferential equations. SIAM J Math Anal 20:116-132

41. Gentili G (1995) Regularity and stability for a viscoelastic material with a singular memory kernel. J Elast 37:139-156

42. Hanyga A (2001) Wave propagation in media with singular memory. Math Comput Model 34:1329-1421

43. Hanyga A (2007) Relations between relaxation modulus and creep compliance in anisotropic linear viscoelasticity. J Elast 88:41-61

44. Hanyga A, Seredynska M (2007) Relations between relaxation modulus and creep compliance in anisotropic linear viscoelasticity. J Elast 88:41-61

45. Hanyga A, Seredynska M (2002) Asymptotic and exact fundamental solutions in hereditary media with singular memory kernels. Q Appl Math 60:213-244

46. Koeller RC (1984) Applications of fractional calculus to the theory of viscoelasticity. ASME J Appl Mech 51:299-307

47. Berti V (2006) Existence and uniqueness for an integraldifferential equation with singular kernel. Boll Un Mat Ital Sez B 9-B:299-309

48. Grasselli M, Lorenzi A (1991) Abstract nonlinear Volterra integrodifferential equations with nonsmooth kernels. (Ital Summ) Atti Accad Naz Lincei Cl Sci Fis Mat Natur Rend Lincei (9) Mat Appl 2(1):4353

49. Giorgi C, Morro A (1992) Viscoelastic solids with unbounded relaxation function. Contin Mech Thermodyn 4:151-165

50. Chepyzhov VV, Mainini E, Pata V (2006) Stability of abstract linear semigroups arising from heat conduction with memory. Asymptot Anal 50:269-291

51. Mainini E, Mola G (2009) Exponential and polynomial decay for first order linear Volterra evolution equations. Q Appl Math 67:93-111

52. Giorgi C, Pata V (2001) Asymptotic behavior of a nonlinear hyperbolic heat equation with memory. Nonlinear Differ Equ Appl 8:157-171

53. Grasselli M, Lorenzi A (1991) Abstract nonlinear Volterra integro-differential equations with nonsmooth kernels. Atti Accad Naz Lincei Cl Sci Fis Mat Natur Rend Lincei (9) Mat Appl 2:43-53

54. Janno J, von Wolfersdorf L (1998) Identification of weakly singular memory kernels in viscoelasticity. Z Angew Math Mech 78:391-403

55. Janno J, von Wolfersdorf L (1997) Identification of weakly singular memory kernels in heat conduction. Z Angew Math Mech 77:243-257

56. Miller RK, Feldstein A (1971) Smoothness of solutions of Volterra integral equations with weakly singular kernels. SIAM J Math Anal 2:242-258

57. Tatar N-E (2009) Exponential decay for a viscoelastic problem with a singular kernel. Z Angew Math Phys 60:640-650

58. Wu ST (2012) Exponential decay for a nonlinear viscoelastic equation with singular kernels. Acta Math Sci $32: 2237-2246$ 
59. Carillo S, Valente V, Vergara Caffarelli G (2010) A result of existence and uniqueness for an integro-differential system in magneto-viscoelasticity. Appl Anal 90:1791-1802

60. Carillo S, Valente V, Vergara Caffarelli G (2012) An existence theorem for the magnetic-viscoelastic problem. Discret Contin Dyn Syst S 5:435-447. doi:10.3934/dcdss. 2012.5.435

61. Carillo S, Valente V, Vergara Caffarelli G (2013) A linear viscoelasticity problem with a singular memory kernel: an existence and uniqueness result. Differ Integral Equ 26(9/ 10):1115-1125

62. Carillo S, Valente V, Vergara Caffarelli G (2014) Heat conduction with memory: a singular kernel problem. Evol Equ Control Theory 3(3):399-410. doi:10.3934/eect.2014.399

63. Carillo S, Valente V, Vergara Caffarelli G (preprint) A 3-dimensional singular viscoelasticity problem

64. Fabrizio M, Gentili G, Reynolds DW (1998) On rigid heat conductors with memory. Int J Eng Sci 36:765-782
65. Amendola G, Carillo S (2004) Thermal work and minimum free energy in a heat conductor with memory. Q J Mech Appl Math 57(3):429-446

66. Carillo S (2005) Some remarks on materials with memory: heat conduction and viscoelasticity. J Nonlinear Math Phys Suppl 1(12):163-178

67. McCarthy M (1970) Constitutive equations for thermomechanical materials with memory. Int J Eng Sci 8:467-474

68. Gentili G (2002) Maximum recoverable work, minimum free energy and state space in linear viscoelasticity. Q Appl Math 60(1):153-182

69. Deseri L, Fabrizio M, Golden JM (2006) A new approach based on the concept of minimal state in viscoelasticity: new free energies and applications to partial differential equations. Arch Rational Mech Anal 181(1):43-96

70. Carillo S, Chipot M, Valente V, Vergara Caffarelli G (2014, preprint) 